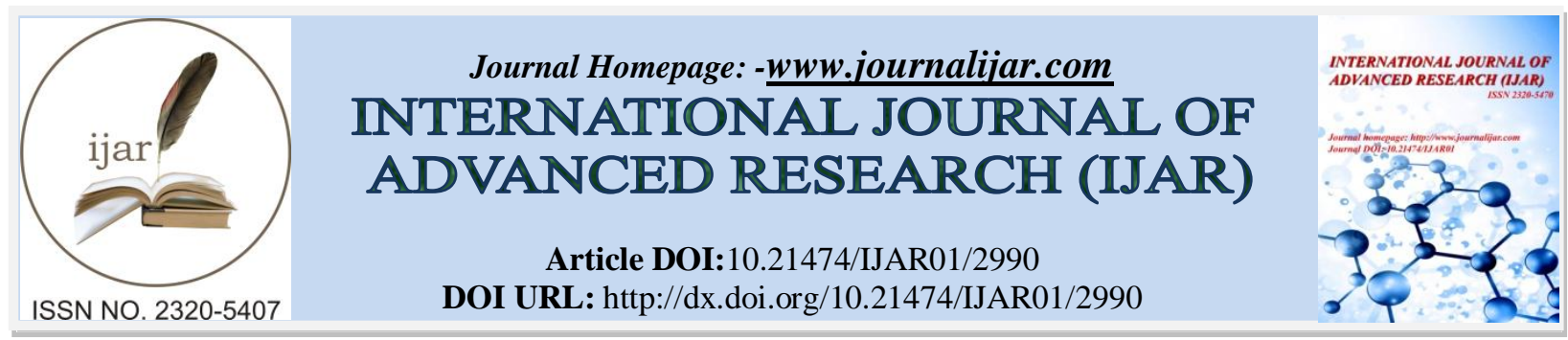

RESEARCH ARTICLE

\title{
A NOVEL APPROACH FOR THE SOLUTION OF A CLASS OF URYSOHN INTEGRALE QUATIONS USING BERNSTEIN POLYNOMIALS.
}

\author{
Jumah Aswad Zarnan. \\ Asst. Prof. Dr., Dept. Of Accounting by IT, Cihan University, Campus, Sulaimaniya, Kurdistan Iraq.
}

Manuscript Info
Manuscript History
Received: 28 November 2016
Final Accepted: 27 December 2016
Published: January 2017
Key words:-
Urysohn-type Fredholm integral
equation, Bernstein polynomials method,
approximate solution.

approximate solution.

\begin{abstract}
In this paper a novel technique implementing Bernstein polynomials is introduced for the numerical solution of a classof Urysohn integral equations. These polynomials are utilized toreduce the solution of the given problem to the solution of a system of non-linear algebraic equations. The remaining set of nonlinear equations is solved numerically by using the approach to yield truncated Bernstein series coefficients of the solution function. Several illustrative examples with numerical simulations are provided to support the theoretical claims.
\end{abstract}

Copy Right, IJAR, 2016,. All rights reserved.

\section{Introduction:-}

The topics of integral equations have been an increasing interest in the past years, because these kinds of equations appear in various fields of applied science and engineering. So, getting solutions with a high level of accuracy for the integral equations is a very important task. Considering that many real-world mathematical problems, especially in the area of applied mathematics are too complicated to be solved in exact terms, the using of numerical methods has been swiftly developed recently. There are many numerical methods for approximating solutions of the linear and non-linear Fredholm integralequations in one and two-dimensionalspaces. In the literature cited below, among the numerous works that have been suggested, some famous approaches are listed as well. Eskandarughlu et al. in [1], presenteda numerical method based on using spline piece-wise functions and Picard's method for solving the Urysohn -type integral equations. The Adomian decomposition method (ADM) for obtaining approximate series solution of Urysohn integral equations was presented in [2]. The numerical approximation solution of the Urysohn integralequationwithtwomethodshasbeenworkedout in [3].

The numerical approximation of this kind of equation has been studied by means of the sinc approximation with the double exponential transformations in [4]. This numerical method combined the sinc Nystrom method with the Newton iterative process that involves solving a nonlinear system of equations. Saberi-Nadjafi and Heidari in [4], offered a combination of the Newton-Kantorovich method and quadrature methods for solving nonlinear integral equations. The method solved the nonlinear integral equations of the Urysohn form in a systematic procedure. Also in $[5,6]$, two iterative schemes based on the homotopy analysis method have been used to the numerical solution of differential equations [7, 8, 9]. On the other hand, the artificial neural networks (ANNs) approach is one of the more applicable methods that have been used for approximating solutions of different kinds of integral equations. For further informationon ANNs in this respectsee $[10,11]$. The ANNs is applied to solve both ordinary and partial differential equations with initial and boundary value problems [12]. In many problems in science and engineering, we have some unknown functions which are too complicated to be determined. The Bernstein polynomials method is one of the earliest analytic-numeric algorithms for approximating the unknown in different kinds of mathematical

Corresponding Author:- Jumah Aswad Zarnan.

Address:-Asst. Prof. Dr., Dept. Of Accounting by IT, Cihan University, Campus, Sulaimaniya, 
problems. This is an extremely useful way of expressing a complicated function in terms of simple polynomials. The only requirement is that the given function should be smooth. In other words, at a point of interest it must be possible to differentiate the function as often as we please.

\section{Bernstein Polynomials:-}

The general form of the Bernstein polynomials [13-16] of nth degree over the interval $[a, b]$ is defined by

$B_{i, n}(x)=\left(\begin{array}{c}n \\ i\end{array}\right) \frac{(x-a)^{i}(b-x)^{n-i}}{(b-a)^{n}}, \quad a \leq x \leq b, \quad i=0,1,2, \ldots, n$

Note that each of these $n+1$ polynomials having degree $n$ satisfies the following properties:

1. $B_{i, n}(x)=0 \quad$ ifi $<0$ ori $>n$

2. $\sum_{i=0}^{n} B_{i, n}(x)=1$

3. $B_{i, n}(a)=B_{i, n}(b)=0, \quad 1 \leq i \leq n-1$

Using MATHEMATICA code, the first 11 Bernstein polynomials of degree ten over the interval $[a, b]$, are given below:

$$
\begin{gathered}
B_{0,10}(x)=(b-x)^{10} /(b-a)^{10} B_{6,10}(x)=210(b-x)^{4}(x-a)^{6} /(b-a)^{10} \\
B_{1,10}(x)=10(b-x)^{9}(x-a) /(b-a)^{10} B_{7,10}(x)=120(b-x)^{3}(x-a)^{7} /(b-a)^{10} \\
B_{2,10}(x)=45(b-x)^{8}(x-a)^{2} /(b-a)^{10} B_{8,10}(x)=45(b-x)^{2}(x-a)^{8} /(b-a)^{10} \\
B_{3,10}(x)=120(b-x)^{7}(x-a)^{3} /(b-a)^{10} B_{9,10}(x)=10(b-x)(x-a)^{9} /(b-a)^{10} \\
B_{4,10}(x)=210(b-x)^{6}(x-a)^{4} /(b-a)^{10} B_{10,10}(x)=(x-a)^{10} /(b-a)^{10} \\
B_{5,10}(x)=252(b-x)^{5}(x-a)^{5} /(b-a)^{10}
\end{gathered}
$$

Now the first six polynomials over $[0,1]$ are shown in Fig. 1(a), and the remaining five polynomials are shown in

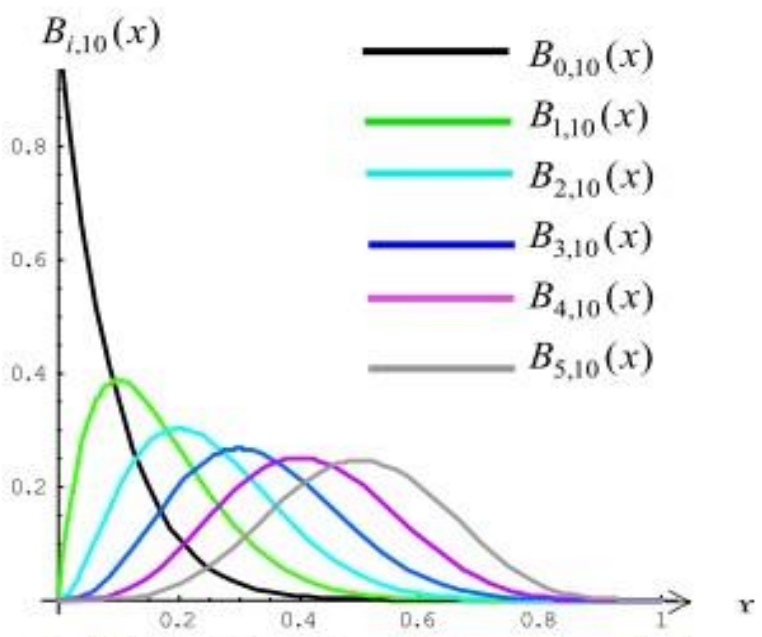

Fig. 1(a): Graph of first 6 Bernstein polynomials over [0, 1]

Fig. 1(b)

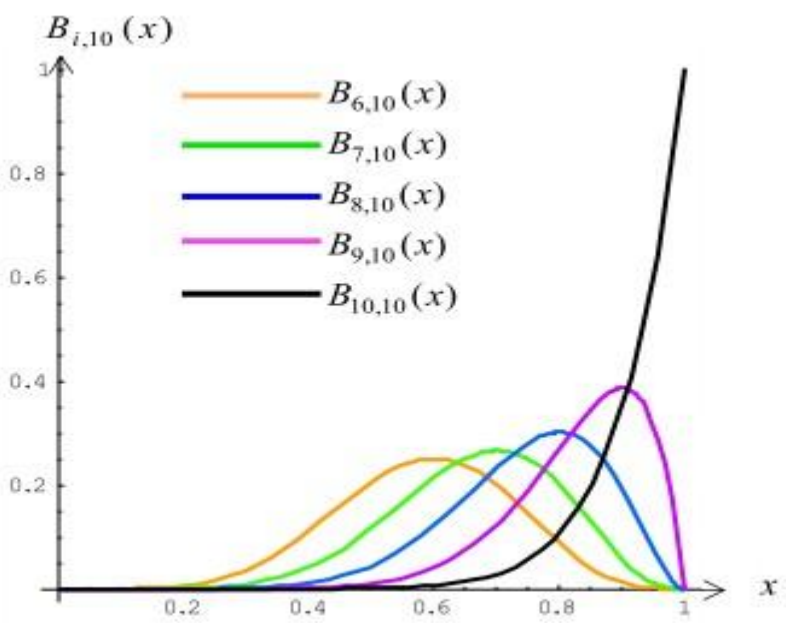

Fig. 1(b): Graph of last 5 Bernstein polynomials over $[0,1]$

\section{Formulation of Integral Equation in Matrix Form:-}

In many applications a matrix formulation for the Bernstein polynomials is useful. These are straight forward to develop if only looking at a linear combination in terms of dot products. Given a polynomial written as a linear combination of the Bernstein basis function [17]:

$$
B(t)=C_{0} B_{0, n}(t)+C_{1} B_{1, n}(t)+\cdots+C_{n} B_{n, n}(t)
$$


It is easy to write this as a dot product of two vectors:-

Which can be converted to the following form:

$$
\left[\mathrm{B}_{0, \mathrm{n}}(\mathrm{t}) \mathrm{B}_{1, \mathrm{n}}(\mathrm{t}) \ldots \mathrm{B}_{\mathrm{n}, \mathrm{n}}(\mathrm{t})\right]\left[\begin{array}{c}
C_{o} \\
C_{1} \\
\vdots \\
C_{n}
\end{array}\right]
$$

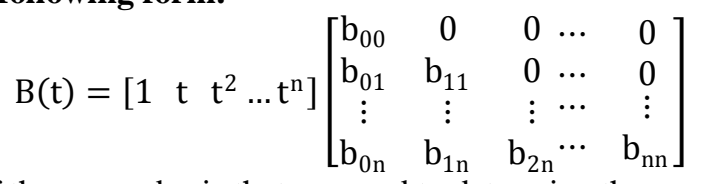

Where $b_{n n}$ are the coefficients of the power basis that are used to determine the respective Bernstein polynomials. We note that the matrix in this case is lower triangular. In the quadratic case(i.e. $n=2)$

The matrix Representation is:-

$$
B(t)=\left[\begin{array}{lll}
1 & \mathrm{t} & \mathrm{t}^{2}
\end{array}\right]\left[\begin{array}{ccc}
1 & 0 & 0 \\
-2 & 2 & 0 \\
1 & -2 & 1
\end{array}\right]\left[\begin{array}{l}
\mathrm{C}_{0} \\
\mathrm{C}_{1} \\
\mathrm{C}_{2}
\end{array}\right]
$$

The cubic case $=3$, the matrix Representation is:-

$$
\mathrm{B}(\mathrm{t})=\left[\begin{array}{lll}
1 & \mathrm{t} & \mathrm{t}^{2} \mathrm{t}^{3}
\end{array}\right]\left[\begin{array}{cccc}
1 & 0 & 0 & 0 \\
-3 & 3 & 0 & 0 \\
3 & 6 & 3 & 0 \\
-1 & 3 & -3 & 1
\end{array}\right]\left[\begin{array}{l}
\mathrm{C}_{0} \\
\mathrm{C}_{1} \\
\mathrm{C}_{2} \\
\mathrm{C}_{3}
\end{array}\right]
$$

\section{Solution of a class of Urysohn integral equations:-}

In this section, first we consider the Urysohn integral equation (UIE) of the second kind given by

$$
x(t)=f(t)+\int_{0}^{1} K(t, s, x(s)) d s
$$

Where $x(t)$ is the unknown functions to be determined, $k(t, s, x(s))$, the kernel is a continuous function, $f(t)$ being the known function. To determine an approximate solution of $(2), x(t)$ is approximated in the Bernstein polynomial basis on $[0,1]$ as:

$$
x(t)=\sum_{i=0}^{n} a_{i} B_{i, n}(t)
$$

Where $a_{i}, i=0,1, \ldots, n$ are unknown constants to be determined using Newton-Raphsonmethod. Substituting (3) in (2), we obtain:

$$
\sum_{i=0}^{n} a_{i} B_{i, n}(t)=f(t)+\int_{0}^{1} K\left(t, s, \sum_{i=0}^{n} a_{i} B_{i, n}(t)\right) d s
$$

Now we put $t=t_{j}, j=0,1, \ldots, n$ in (4), $t_{j}$ 's being chosen as suitable distinct points in $[0,1]$, such that $t_{0}=0$, $t_{n}=1$, and $t_{j}=t_{0}+j h$, where $h=(1-0) / n$. Puttingt $=t_{j}$, we obtain the nonlinear system:

$$
\sum_{i=0}^{n} a_{i} B_{i, n}\left(t_{j}\right)=f\left(t_{j}\right)+\int_{0}^{1} K\left(t_{j}, s, \sum_{i=0}^{n} a_{i} B_{i, n}\left(t_{j}\right)\right) d s
$$

The nonlinear system (5) can be solved by standard methods for the unknown constant $a_{I}$ 's . These $a_{i}, i=$ $0,1, \ldots, n$ are then used in (3) to obtain the unknown function $x(t)$ approximately.

The following algorithm summarizes the steps for finding the approximate solution for the second kind of nonlinear Urysohn integral equation. 


\section{Algorithm (BPNUIE):}

Step (1):

Assume $\mathrm{t}_{0}=0, \mathrm{t}_{\mathrm{n}}=1$, and $\mathrm{t}_{\mathrm{j}}=\mathrm{t}_{0}+\mathrm{jh}$, where $\mathrm{h}=(1-0) / \mathrm{n}$.

Step (2):

Putting $\mathrm{t}=\mathrm{t}_{\mathrm{j}}$ in (4) to obtain nonlinear System (5).

Step (3):

Solved the nonlinear system (5) to calculate the unknowna $a_{i}, i=1, \ldots, n$.

Step (4):

Used $a_{I}$ in (3) to obtain the function $\mathrm{x}(\mathrm{t})$ approximately.

\section{Numerical Examples:-}

In this section, the method presented in this paper is used to find numerical solution of two illustrative examples. The solution of the equations obtained here. All calculations in the following tables are performed using Matlab.

Example1. [4] Let us solve the Urysohn integral equation:

$$
\int_{0}^{1} \cos (\pi x) \sin (\pi t) y 3(t) d t=5(y(x)-\sin (\pi x)), \quad 1 \leq x \leq 1,
$$

By the explained Bernstein series method. The exact solution corresponding to this equationis $y(x)=$ $\sin (\pi x)+1 / 3(20-\sqrt{391}) \cos (\pi x)$.

For $\mathrm{n}=4$, theoriginalintegral equation is reduced to a fundamental non-linear system. The iterative process yields the results which have been gathered in Table 1. Figure 2 shows the exact solution and the approximate solution.

Table 1:-Numerical results for Example 1.

\begin{tabular}{|c|c|c|c|}
\hline $\mathrm{si}=0.1 \mathrm{i}$ & Exact solution & Approximatesolution & Error \\
\hline $\mathrm{i}=0$ & 0.0754266889049 & 0.0748255 & $6.01 \mathrm{E}-04$ \\
\hline $\mathrm{i}=1$ & 0.3807520383605 & 0.3810056 & $2.54 \mathrm{E}-04$ \\
\hline $\mathrm{i}=2$ & 0.6488067254460 & 0.6435965 & $5.21 \mathrm{E}-03$ \\
\hline $\mathrm{i}=3$ & 0.8533516897425 & 0.8498055 & $3.55 \mathrm{E}-03$ \\
\hline $\mathrm{i}=4$ & 0.9743646449962 & 0.9702287 & $4.14 \mathrm{E}-03$ \\
\hline $\mathrm{i}=5$ & 1 & 0.9702355 & $8.98 \mathrm{E}-02$ \\
\hline $\mathrm{i}=6$ & 0.9277483875941 & 0.9188357 & $1.41 \mathrm{E}-03$ \\
\hline $\mathrm{i}=7$ & 0.7646822990073 & 0.7632754 & $6.21 \mathrm{E}-03$ \\
\hline $\mathrm{i}=8$ & 0.5267637791389 & 0.5205584 & $5.86 \mathrm{E}-03$ \\
\hline $\mathrm{i}=9$ & 0.2372819503893 & 0.2314202 & $2.23 \mathrm{E}-03$ \\
\hline $\mathrm{i}=10$ & -0.0754266889049 & -0.0732011 & \\
\hline
\end{tabular}




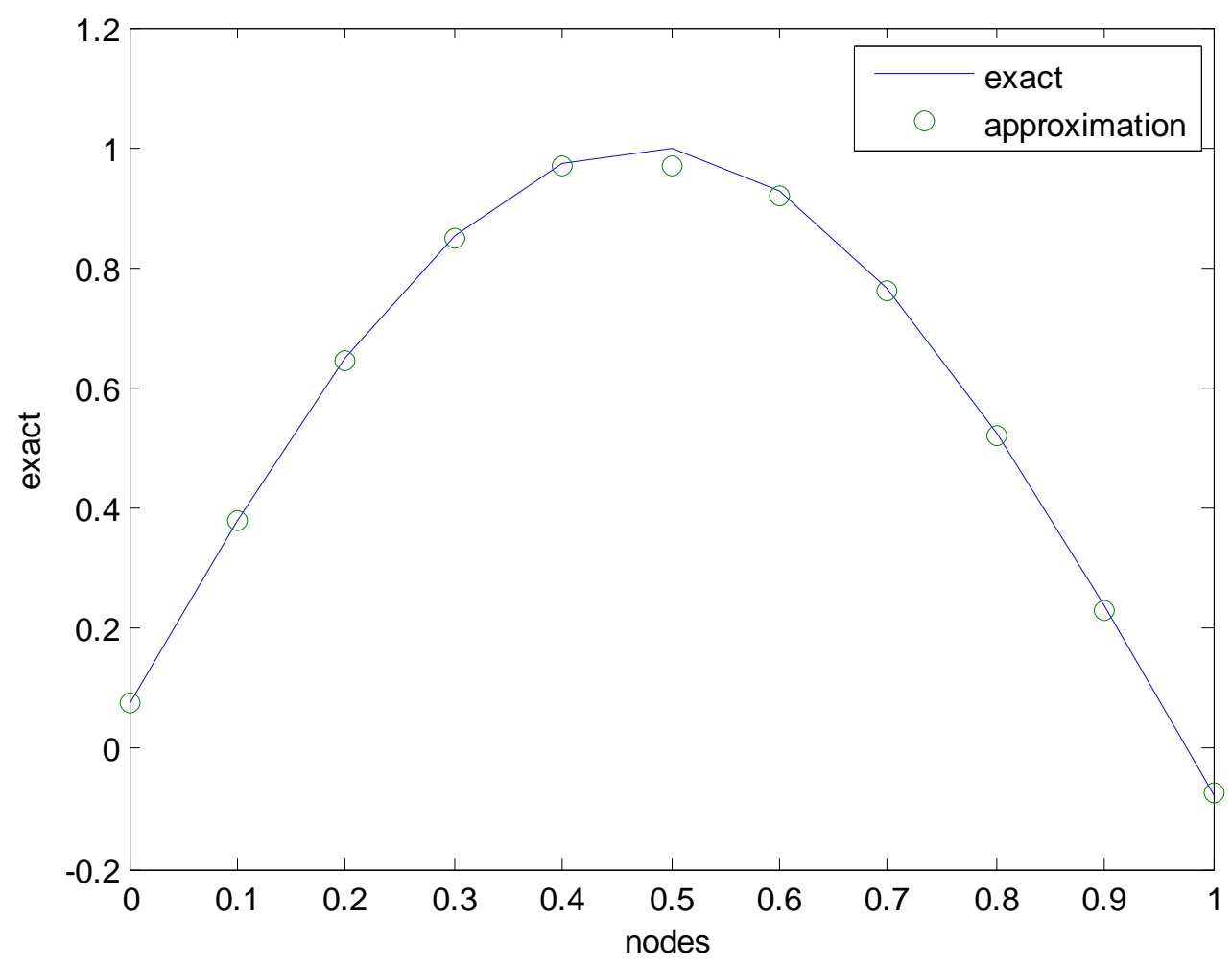

Fig. 2:- Comparison of the exact and approximate solutions for Example 1.

Example2. [5] Consider the Urysohn integral equation:

$$
\int_{0}^{1}(x-t) y^{2}(t) d t=y(t)-\ln \left(4(x+1)(1-x \ln 2+x)^{2}\right)+2 x+\frac{5}{4}
$$

With the exact solution $y(x)=\ln (x+1)$ and use the present method for finding its approximate solution. The iterative process yields the results which have been gathered in Table 2. Figure 3 shows the exact solution and the approximate solution.

Table 2:-Numerical results for Example 2.

\begin{tabular}{|c|c|c|c|}
\hline $\mathrm{si}=0.1 \mathrm{i}$ & Exact solution & Approximatesolution & Error \\
\hline $\mathrm{i}=0$ & 0 & 0.0010088 & $1.01 \mathrm{E}-03$ \\
\hline $\mathrm{i}=1$ & 0.095310179804 & 0.0953524 & $4.22 \mathrm{E}-05$ \\
\hline $\mathrm{i}=2$ & 0.182321556793 & 0.1822203 & $1.01 \mathrm{E}-04$ \\
\hline $\mathrm{i}=3$ & 0.262364264467 & 0.2621755 & $1.89 \mathrm{E}-04$ \\
\hline $\mathrm{i}=4$ & 0.336472236621 & 0.3363668 & $1.05 \mathrm{E}-04$ \\
\hline $\mathrm{i}=5$ & 0.405465108108 & 0.4055742 & $1.09 \mathrm{E}-04$ \\
\hline $\mathrm{i}=6$ & 0.470003629245 & 0.4713763 & $3.37 \mathrm{E}-03$ \\
\hline $\mathrm{i}=7$ & 0.530628251062 & 0.5310079 & $5.88 \mathrm{E}-04$ \\
\hline $\mathrm{i}=8$ & 0.587786664902 & 0.5871986 & $4.64 \mathrm{E}-04$ \\
\hline $\mathrm{i}=9$ & 0.641853886172 & 0.6413895 & $1.97 \mathrm{E}-04$ \\
\hline $\mathrm{i}=10$ & 0.693147180559 & 0.6929504 & \\
\hline
\end{tabular}




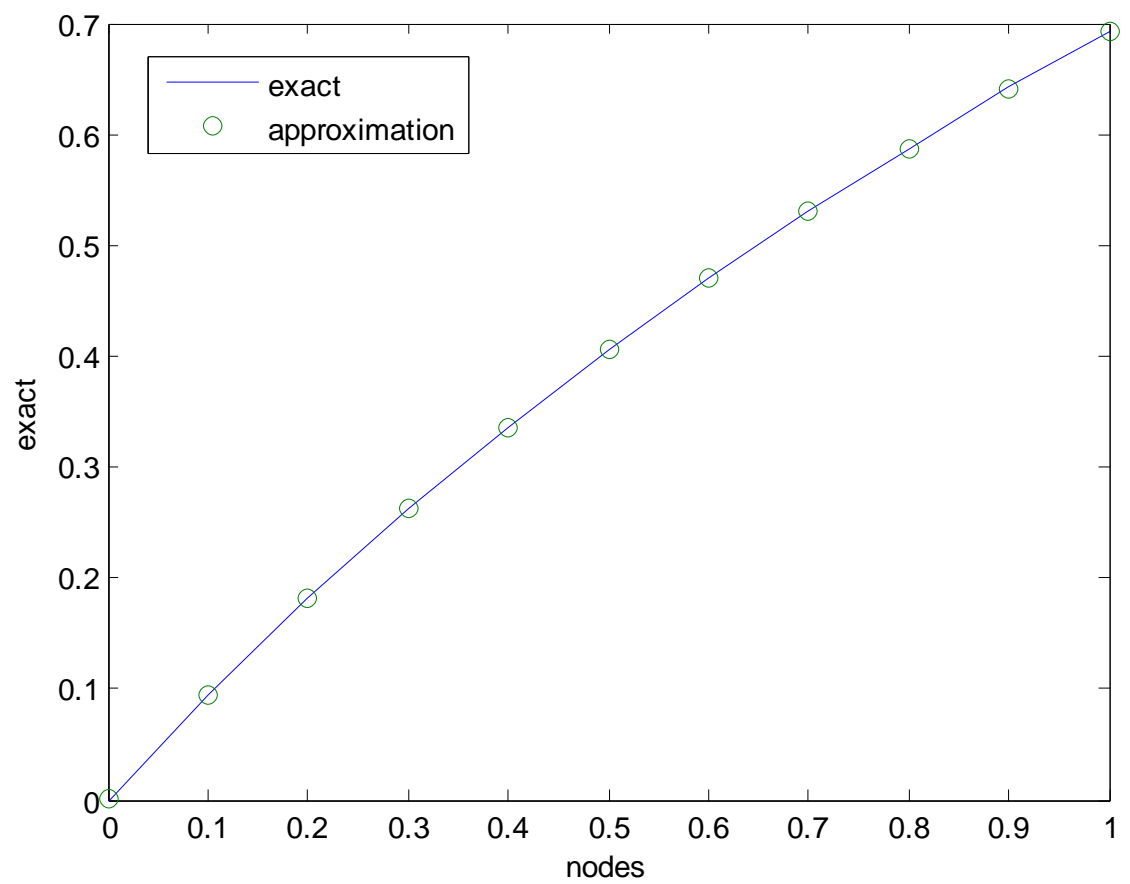

Fig. 3:- Comparison of the exact and approximate solutions for Example 2.

It follows from the results of these examples that $y_{n}(x)$ converge as $n \rightarrow \infty$ to the exact solution $y(x)$ of the integral equation. A successful choice of the "zeroth" approximation can result in a rapid convergence of the procedure.

\section{Conclusion:-}

In this paper, we presented a useful numerical method that originated mainly from the Bernstein polynomials for solving Urysohn type integral equation. As we explained above, this method converts the present problem to a system of non-linear algebraic equations which may not be solvable easily. Having determined the unknown Bernstein coefficients of the solution function, the series solution is produced for numerical purposes immediately. It is important to be noted that, the more terms must be evaluated to the higher accuracy level. The obtained numerical results from analyzed examples illustrated that in applications involving computations with polynomials, the Bernstein form offers an efficient algorithm for many basic functions.

\section{References:-}

1. M. Eskandarughlu, H.E. Derili Gherjalar, H. Mohammadikia, A. Arzhang, Austra. J. Basic Appl. Sci.7 (1),

2. 286-294 (2013).

3. R. Singh, G. Nelakanti, J. Kumar, the Sci. World J. 2014, Article ID 150483 (2014).

4. K. Maleknejad, K. Nedaiasl, J. Int. Equ. Appl. 25(3), 321-454 (2013); K.Maleknejad, K.Nedaiasl, B.Moradi,ProceedingsoftheWorldCongressonEngineering,2013, Vol I.

5. J. Saberi-Nadjafi, M. Heidari, Comput. Math.Applications 60, 2058-2065 (2010).

6. A. Jafarian, Z. Esmailzadeh, L. Khoshbakhti, Appl. Math. Sci.7(28), 1375-1385 (2013).

7. F. Awawdeh, A. Adawi, Int. Math. Forum17, 805-817 (2009).

8. A.K. Golmankhaneh, N.A. Porghoveh, D. Baleanu, Rom. Rep. Phys. 65, 350-361 (2013);

9. D. Rostamy, M. Alipour, H. Jafari, D. Baleanu, Rom. Rep. Phys. 65, 334-349 (2013);

10. A.K. Golmankhaneh, Ali K. Golmankhaneh, D. Baleanu, Rom. Rep. Phys.63, 609-623 (2011);

11. A.K. Golmankhaneh, Ali K. Golmankhaneh, D. Baleanu, Sig. Proce. 91(3), 446-451 (2011).

12. A. Kadem, D. Baleanu, Rom. J. Phys. 56, 332-338 (2011);

13. X.J. Yang, D. Baleanu, W.P. Zhong, Proc. Romanian Acad. A14, 127-133 (2013).

14. A.M.O. Anwar, F. Jarad, D. Baleanu, F. Ayaz, Rom. J. Phys.58, 15-22 (2013); Jafarian et al., Rom. J. Phys.58, 694-702 (2013);

15. J.J. Rosales Garcia, M.G. Calderon, J.M. Ortiz, D. Baleanu, Proc. Romanian Acad. A 14, $42-47$ (2013). 
16. A. Jafarian, S. Measoomy Nia, J. Hyperstruct. 2(1), 53-71 (2013);Jafarian, S. Measoomy Nia, Appl. Math. Model. 37(7), 5027-5038 (2013); Jafarian, S. Measoomy Nia, Int. J. Math. Mod.Numer. Opt.n 4(3), 225-237 (2013);

17. Jafarian, S. Measoomy Nia, A.K. Golmankhaneh, D. Baleanu, Adv. Diff. Equ. DOI:10.1186/1687-1847-2013123.

18. S. Effati, R. Buzhabadi, Neural Comput. Appl., DOI:10.1007/s00521-010-0489-y.

19. I.E. Lagaris, A. Likas, D.I. Fotiadis, IEEE Transac. Neur. Netw. 9(5), 987-1000 (1998).

20. J. Reinkenhof, Int. J. Numer. Methods Engrg. 11, 1627 (1986).

21. E. Kreyszig, Int. J. Numer. Methods Engrg.14, 292 (1979).

22. B. N. Mandal and S. Bhattacharya, Appl. Math.Comput. 190, 1707 (2007).

23. M.I. Bhatti and P. Bracken, J. Comput.Appl.Math.205, 272 (2007).

24. Muna M. Mustafa \& Khawla A .AL-Zubaidy. Use of Bernstein Polynomial in Numerical Solution of Nonlinear Fred Holm Integral Equation. Eng. \& Tech. Journal, Vol. 29, No. 1, 2011 